\title{
Revisión bibliométrica de COVID-19 en el periodo diciembre 2019 y julio 2020
}

\section{A bibliometric review of COVID-19 from december 2019 to july 2020}

\author{
Luis Eduardo Pino-Villarreal, Iván Camilo Triana-Avellaneda, Mateo Barros-Barraza, \\ lucía Carolina Viola-Muñoz • Bogotá, D.C. (Colombia)
}

DOI: https://doi.org/10.36104/amc.2020.1879

\section{Resumen}

Objetivo: realizar el análisis bibliométrico de COVID-19 durante diciembre de 2019 a 30 de junio de 2020.

Métodos: revisión bibliométrica del total de la literatura y de lo relacionado con COVID-19 en las bases Pubmed, Scopus y Lilacs durante el periodo diciembre de 2019 a 30 junio de 2020. Se clasificaron los artículos por categorías (objetivo, país, especialidad) y se compararon con la literatura del año anterior.

Resultados: se encontraron 27 373, 16944 y 1083 publicaciones acerca de COVID-19 en Pubmed, Scopus y Lilacs respectivamente. La principal especialidad médica por búsqueda fue neumología. De acuerdo con el objetivo, el más encontrado fue tratamiento (50.0\%). El país con más publicaciones fue Estados Unidos (28.9\%) en Pubmed y Scopus. En el lapso COVID-19 las publicaciones de la enfermedad representan 0.06 del total.

Conclusiones: existe un volumen importante de publicaciones relacionadas con COVID-19 en periodo de estudio, equivalente a $6 \%$ de las publicaciones totales, lo cual es significativo para una sola enfermedad. (Acta Med Colomb 2020; 45. DOI: https://doi.org/10.36104/amc.2020.1879).

Palabras clave: COVID-19, revisión bibliométrica, estadística descriptiva.

\section{Abstract}

Objective: to perform a bibliometric analysis of COVID-19 from December 2019 to June 30, 2020.

Methods: a bibliometric review of all the literature and COVID-19 related material in the PubMed, Scopus and Lilacs databases from December 2019 to June 30, 2020. Articles were classified by categories (objective, country, specialty) and compared to the previous year's literature.

Results: a total of 27,373,16,944 and 1,083 publications on COVID-19 were found in PubMed, Scopus and Lilacs, respectively. The main medical specialty by search was pulmonology. The most frequently found objective was treatment $(50.0 \%)$. The country with most publications was the United States (28.9\%) on PubMed and Scopus. During the COVID-19 span, publications on the disease represented 0.06 of the total.

Conclusions: there was a significant volume of COVID-19 related publications during the study period, equivalent to $6 \%$ of the total publications, which is significant for a single disease. (Acta Med Colomb 2020; 45. DOI: https://doi.org/10.36104/amc.2020.1879).

Key Words: COVID-19, bibliometric review, descriptive statistics.
Dr. Luis Eduardo Pino-Villarreal: Hematólogo y Oncólogo Clínico. Magíster en Oncología Molecular y en Administración de la Salud MBA. Departamento de Oncología Hospital Universitario Fundación Santa Fe de Bogotá, Facultad de Medicina, Universidad de los Andes; Dr. Iván Camilo Triana-Avellaneda: Asistente de Investigación en Oncología, Departamento de Oncología, Hospital Universitario Fundación Santa Fe de Bogotá; Mateo Barros-Barraza: Estudiante de Medicina, Facultad de Medicina Universidad de los Andes; Dra. Lucía Carolina Viola-Muñoz: Especialista en Medicina Interna y Neumología, Departamento de Oncología Fundación Neumológica Colombiana. Bogotá, D.C. (Colombia).

Correspodencia: Dr. Iván Camilo TrianaAvellaneda. Bogotá, D.C. (Colombia).

E-mail: camilotrianaa@gmail.com

Recibido: 15/V/2020 Aceptado: 17/VII/2020

\section{Introducción}

En diciembre de 2019 se presentó el primer caso conocido de infección por un nuevo coronavirus originado en la ciudad de Wuhan, China (1). El 31 de diciembre del mismo año, la oficina regional de la Organización Mundial de la Salud (OMS) desde su sede en Beijing reportó un clúster de casos similares de infección respiratoria, puntualmente en la misma ciudad de Wuhan. Todos los casos documentados tenían el antecedente común de contacto con el mercado de mariscos de la ciudad.

El 8 de enero del 2020 el Centro para Prevención y Control de Enfermedades (CDC) en su primer boletín, describió 
la investigación de casos en China de infección respiratoria aguda. A la fecha del reporte no se habían identificado casos por fuera del país asiático.

En el informe de la OMS de enero 20 de 2020 se reportaron cuatro por fuera de China en Tailandia, Japón y Corea, todos ellos correspondían a casos exportados desde Wuhan. De los 278 reportes propios de China, se informaban seis muertes por la enfermedad (2).

Se identifica un nuevo coronavirus en muestras de lavado broncoalveolar de pacientes con la infección respiratoria aguda, mediante técnica de reacción en cadena de la polimerasa (PCR) en tiempo real se identifica ácido nucleico viral. De igual forma en ácido ribonucleico (RNA) extraído del lavado broncoalveolar (LBA) es posible realizar la secuenciación genética (2). El nuevo coronavirus, germen causal de la infección identificado en tres pacientes provenientes de Wuhan, con cuadro clínico compatible, corresponde a un betacoronavirus, subgénero sarbecovirus de la familia coronaviridae. Con técnicas similares, poco tiempo después se confirman estos hallazgos con nueve casos más, lo que a su vez permitió el desarrollo de una prueba por técnica de PCR para identificación de la infección (3).

Tres meses después de las primeras descripciones, la enfermedad corresponde hoy a una pandemia, que se ha diseminado en todos los continentes, a excepción de la Antártica.

En Colombia a la fecha, se conocen más de 150000 casos con casi 6000 fallecidos (corte julio 14/2020). Poco se conoce sobre el comportamiento clínico por fuera de los casos inicialmente informados en China, la dispersión del virus en otros países no ha tenido las mismas características, en particular en Italia (4) y aún hoy no existe tratamiento específico para la infección.

El personal de salud a cargo del cuidado de los pacientes infectados se encuentra en un alto riesgo de contraer la enfermedad, similar a la situación presentada en epidemias previas de coronavirus similares como la de síndrome respiratorio agudo severo (SARS) en 2002-2003 en Hong Kong, Toronto, Singapur y Guangzhou (5).

No existen estrategias claras de prevención de la infección desde el punto de vista farmacológico. Las intervenciones no farmacológicas que han demostrado reducción en la transmisibilidad de la infección son el lavado e higiene de manos, el aislamiento social, la cuarentena obligatoria en los viajeros a zonas de riesgo o posterior al contacto con infectados sin protección y el cierre de los lugares donde pueda existir alta congregación de personas (escuelas, teatros, eventos deportivos) (6).

Al ser declarado en marzo de 2020 como una pandemia por la OMS, la enfermedad COVID-19 ha llevado a enfocar las capacidades científicas de los diferentes países casi que exclusivamente al estudio de esta enfermedad. De igual forma globalmente se han implementado medidas de distanciamiento social destinadas a mitigar el impacto de la pandemia. A pesar de llevar solamente cuatro meses en el escenario científico el virus SARS-CoV-2 está generando datos a una velocidad abismal con diversas publicaciones de toda índole incluyendo ensayos clínicos de rápido desarrollo en busca de intervenciones terapéuticas efectivas y vacunas. Es importante conocer el impacto bibliométrico de la pandemia para conocer el volumen de información publicada, los tópicos cubiertos, los países que están produciendo esta literatura y el potencial impacto generado por la desviación de prioridades hacia esta enfermedad, como un costo de oportunidad para la investigación en otros temas de la medicina.

\section{Metodología}

Se realizó una revisión bibliométrica desde diciembre $1^{\circ}$. de 2019 hasta el 30 de junio de 2020 (fecha de corte del artículo). Para ellos se consultaron las bases de datos: Pubmed, Scopus y Lilacs (Literatura latinoamericana y del Caribe en Ciencias de la Salud) para la búsqueda de los artículos según los criterios de búsqueda especificados. Se filtraron todos los artículos publicados con las palabras clave: "COVID-19" y/o SARS-CoV-2, el término de búsqueda fue "COVID-19 or 2019-novc". Los artículos encontrados fueron clasificados por especialidad, por el objetivo focal del artículo, por el país de origen de la publicación y por la revista de publicación. En paralelo se hizo una revisión desde diciembre de 2018 hasta el 30 de junio de 2019 de los diferentes artículos de medicina publicados en las mismas bases de datos para tener un comparativo inicial del universo de publicaciones fuera del escenario COVID-19. Para el análisis de datos se utilizó la plataforma MAIA data (plataforma propia) y el programa VOSviewer.

\section{Resultados}

En el periodo de $1^{\circ}$. de diciembre de 2019 a 30 de junio de 2020 se encontraron un total de 45400 artículos sobre COVID-19 y/o SARS-CoV-2 para las tres bases de datos. En la Tabla 1 se muestra la distribución de estos artículos según la base correspondiente así: Pubmed 27373 artículos, Scopus 16944 y Lilacs 1083 artículos.

En el periodo del $1^{\circ}$. de diciembre de 2018 a 30 de junio de 2019 se encontraron 743175 artículos de toda la literatura médica para dos bases (Pubmed y Lilacs), dado que

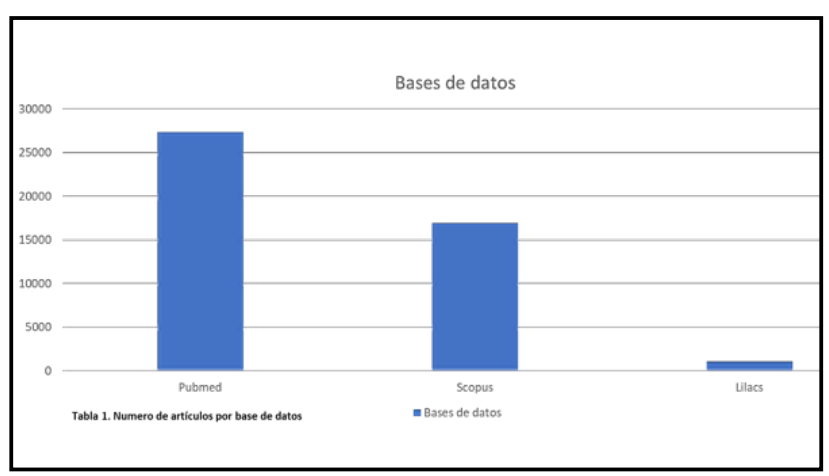

Tabla 1. Número de artículos por base de datos. 


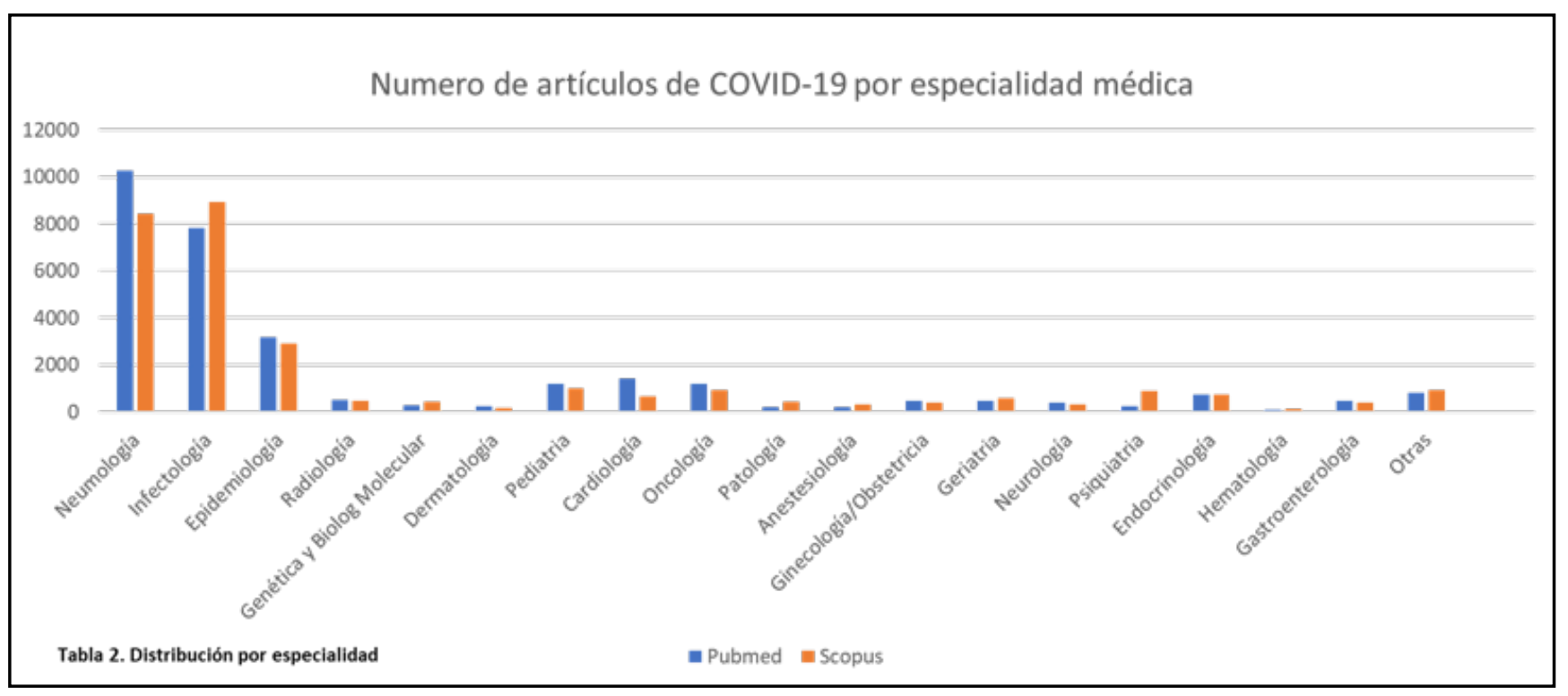

Tabla 2. Distribución por especialidad.

en Scopus no se pudo realizar filtro por periodo de meses específicos. Al comparar el periodo pre COVID-19 (20182019) con el periodo COVID-19 (2019-2020) se encuentra que hay una diferencia de 57747 en el total de publicaciones de la literatura médica en las bases de Pubmed y Lilacs, evidenciando un aumento de $7 \%$ en las publicaciones del periodo COVID-19. Al evaluar los artículos de COVID-19 en los dos periodos se encuentra que en el periodo pre COVID-19 los artículos relacionados con este tema son de $0 \%$ (la enfermedad no estaba descrita), pero en el lapso COVID-19 las publicaciones de la enfermedad representan 0.06 del total de lo publicado, visto en porcentaje representan $6 \%$ de las publicaciones totales en el periodo diciembre del 2019 a 30 de junio del 2020.

En la Tabla 2 podemos ver cómo se han concentrado los artículos de las bases de datos Pubmed y Scopus según la especialidad médica, no se realizó esta clasificación en Lilacs por la naturaleza de los artículos y en la Tabla 3 vemos los principales objetivos o intenciones de los artículos que se han realizado sobre COVID-19.

Se realizó también la distribución de los artículos según cada país, resultados que se encuentran en la Tabla 4. Mediante el programa VOSviewer se correlacionaron las palabras claves de los artículos con un mínimo de dos ocurrencias, se extrajeron las correlaciones de las "keywords" de los artículos en las Figuras 1 y 2, se reporta la correlación de términos reportados en el abstract, de esta forma se reporta visualmente cuáles son los términos o conceptos en los que están centrados las investigaciones de COVID-19. Por otro lado, se realizó la correlación de las revistas en donde se han publicado los artículos, con un mínimo de dos ocurrencias encontrando los resultados de la Figura 3.

La distribución por país se realizó en las tres bases de datos, con 19192 artículos en Pubmed que no se pudieron

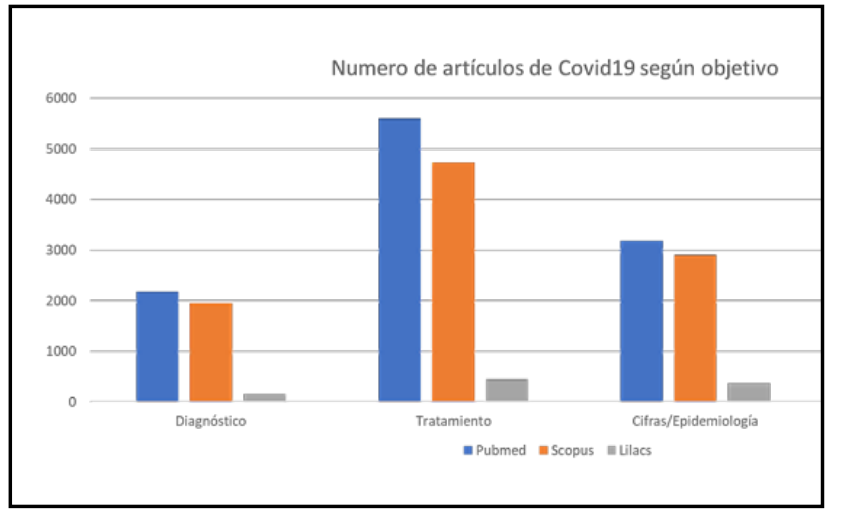

Tabla 3. Distribución según objetivo.

clasificar. Se encontró que los países con mayor volumen de publicación fueron Estados Unidos con 28.9\%, Inglaterra $17.2 \%$, seguido de China con $12.7 \%$ e Italia $10.1 \%$. En Lilacs base latinoamericana, los países con mayor participación fueron Brasil $43.5 \%$, Colombia $28.7 \%$ y Perú $10.3 \%$

\section{Discusión}

La presente revisión bibliométrica muestra un crecimiento incremental de publicaciones relacionadas con COVID-19 en el primer semestre de 2020 contabilizando el $6 \%$ del total de publicaciones médicas para tres de las principales bases de revistas tanto globales como latinoamericanas, pero llevando a un crecimiento comparativo del $7 \%$; lo cual es significativo para una sola enfermedad. En un reporte no publicado previamente por nuestro grupo, con corte a 31 de marzo de 2020 había 0.005 de publicaciones correspondientes a esta enfermedad, es decir, $0.5 \%$ comparado con $6 \%$ a corte de junio 30 de 2020 , es decir, tres meses después observamos un crecimiento de $1200 \%$ que corresponde a 12 veces el corte del primer trimestre del mismo año. 
Distribución por país

25000

20000

15000

10000

5000

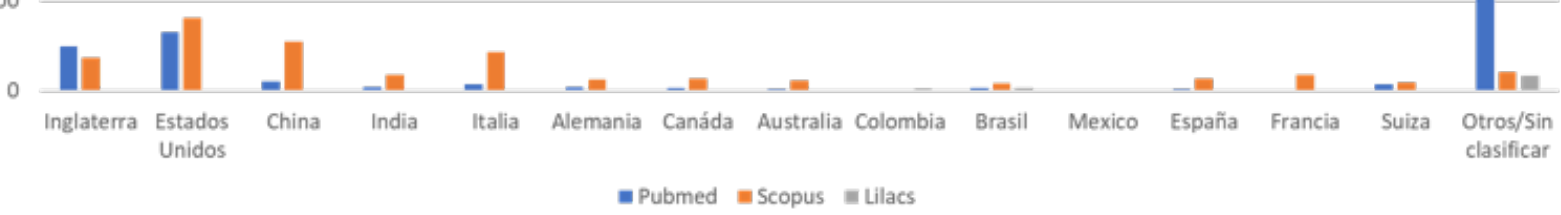

Tabla 4. Distribución por país.

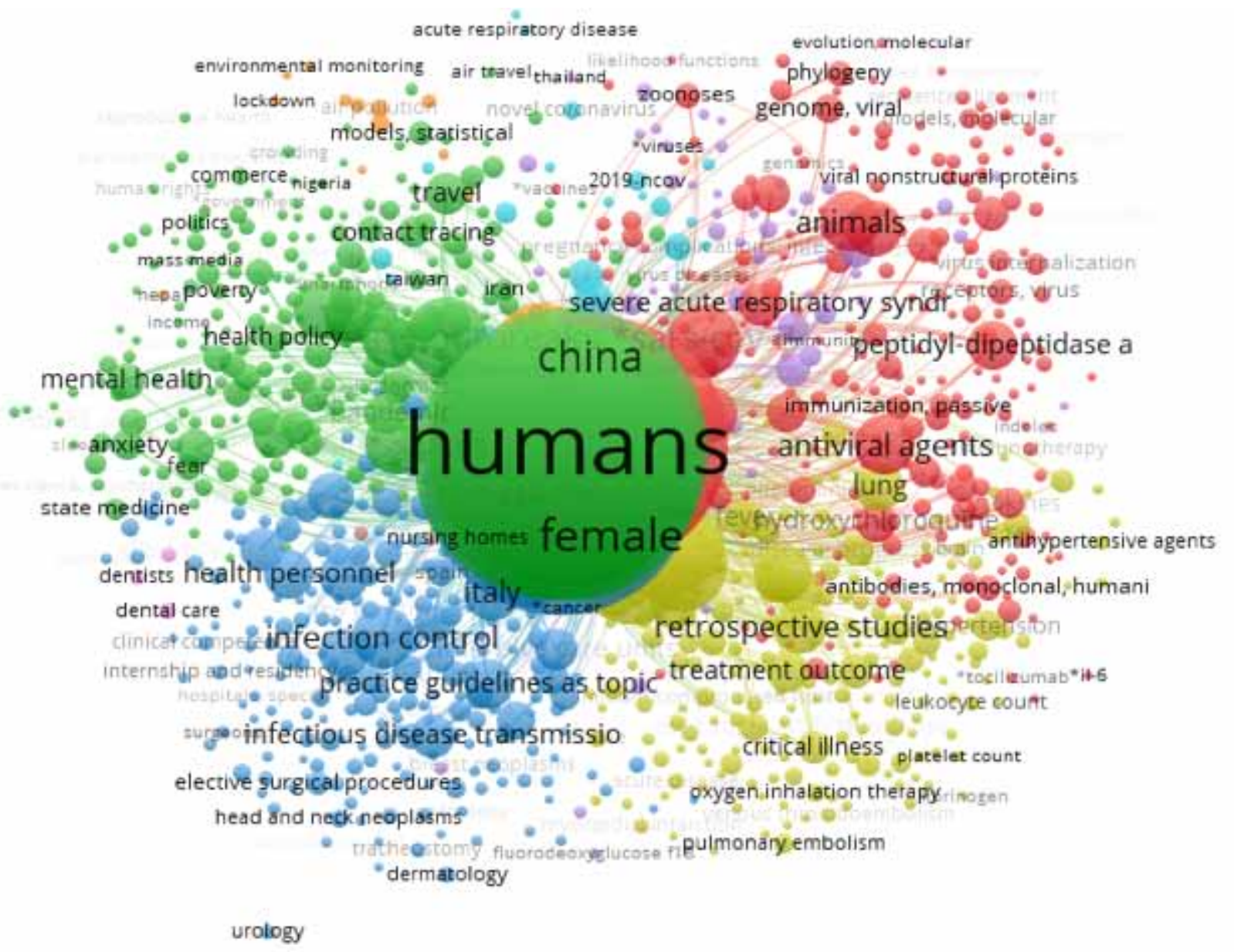

Figura 1. Correlación keywords. Las líneas que interconectan círculos representan las 100 correlaciones más repetidas. 


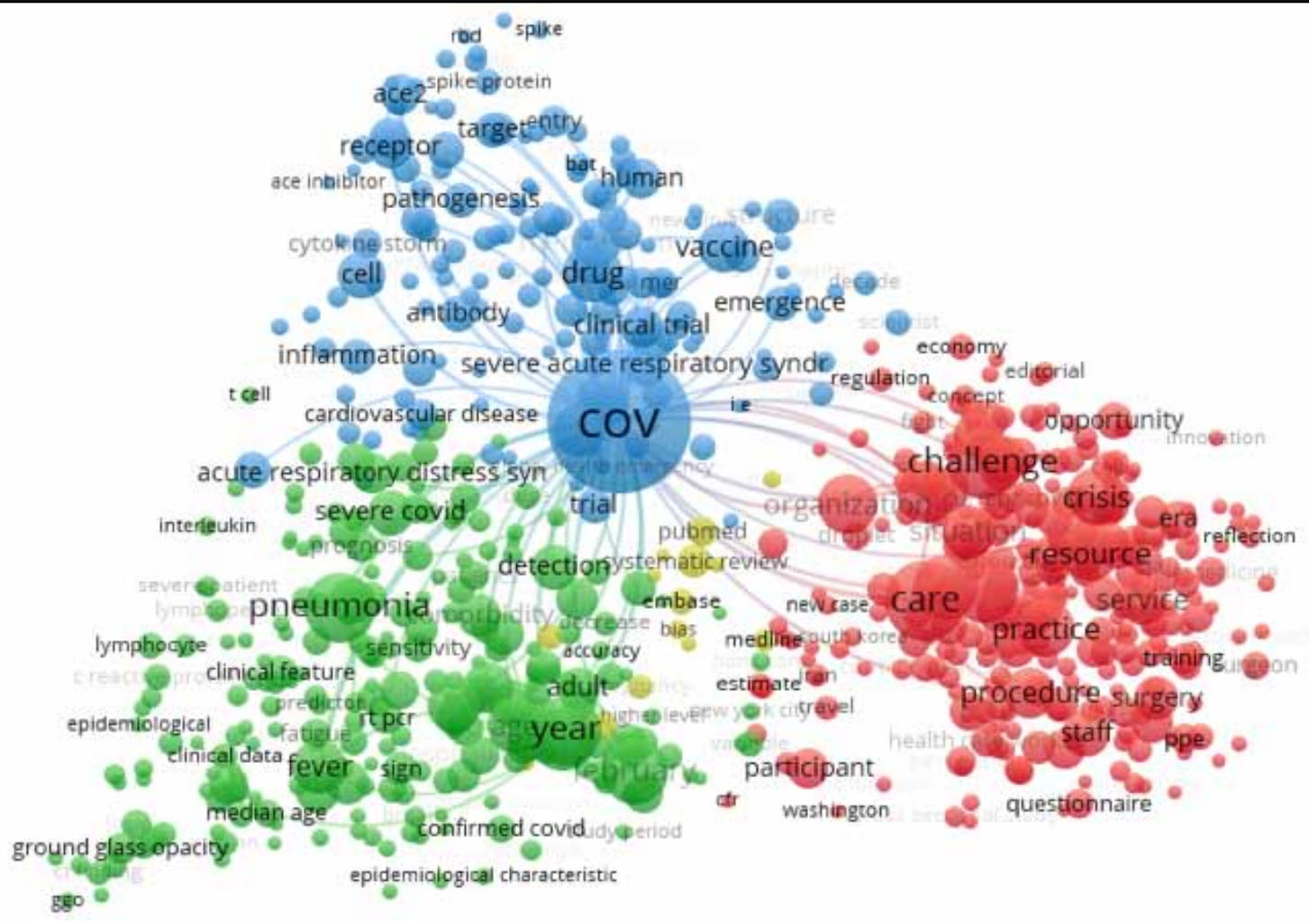

Figura 2. Correlación de términos en abstract. Las líneas que interconectan círculos representan las 100 correlaciones mas repetidas.

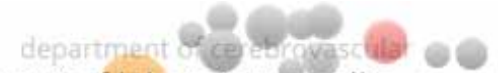 \\ department of laboratory mediepartment of biomedical scien \\ C. department of gastroenterology}

school of nursing, hong kong $p$

internatonalscientific shifpgzhou eighth pegple's hosp aix marseille universite. ird.

ucl medical school, university department of efletiobjogy th

diabetes foundation (india), $\mathrm{n}$ e ervergency and tyauma surgeryyong loa lin school of medicin department afgirical labgrats hubel province key laboratpry london school of hygiene and clinical immunology unit, depa department of medical, surgica

department of internal medicin
these authors corntributed equa

college of medicine and public

department of general surgery.

school of medicine, college of

department of pulmonary and cr

institute of clinical sciences

$$
\begin{aligned}
& \text { servicio deurologia. hospital dr dy patil university, pune, } \\
& \text { laboratory tor in đustrialarescola nacional de saúde públi } \\
& \text { department of biochemistry.pr }
\end{aligned}
$$

Figura 3. Correlación de journals de publicación. La grafica muestra los journals con mínimo dos ocurrencias en la literatura. 
Respecto a los tópicos específicos los tres en los cuales se han publicado mayor cantidad de artículos han sido neumología, infectología y epidemiología; éstos se correlacionan con los principales términos de búsqueda que han sido humanos, control de infección, agentes retrovirales. De igual forma se encuentra una distribución heterogénea de las revistas o lugares de publicación de los artículos.

Respecto a la contribución de publicaciones por países es claro el liderazgo por parte de Estados Unidos en donde se concentra la investigación referente al virus y de China en donde se dieron los primeros informes de reporte de casos al ser el país de origen del brote. En Latinoamérica por otra parte Brasil y Colombia lideran las publicaciones, pero estas principalmente han sido protocolos y guías de tratamiento de la enfermedad, así como modelaciones epidemiológicas.

El virus SARS-CoV-2 y su enfermedad COVID-19 han tenido un impacto importante en las publicaciones médicas la cual se verá posiblemente de mayor impacto en el segundo trimestre de 2020. Esto demuestra la capacidad de reacción de la comunidad científica ante esta amenaza de salud pública, pero a la vez permite concluir que esta primera fase estuvo jalonada principalmente por artículos relacionados con la fisiopatología del virus y las modelaciones estadísticas, con un peso importante sobre el total de la literatura médica. Es posible que en fases posteriores se publiquen datos más relacionados con terapéutica, salud global y epidemiología. $\mathrm{Al}$ ser este un tema en constante evolución es importante dar continuidad a los estudios bibliométricos para conocer las tendencias de publicación ya que las revistas de alto impacto están siendo colmadas de solicitudes de publicación y algunas de ellas han optado por aceptar tan sólo un 16 a $20 \%$ de los artículos recibidos (dato recibido por el grupo por parte de una revista internacional).

\section{Referencias}

1. Zhu N, Zhang D, Wang W, Li X, Yang B, Song J, et al. A novel coronavirus from patients with pneumonia in China, 2019. N Engl J Med. 2020;382(8):727-33.

2. World Health Organization (WHO). Novel Coronavirus (2019-nCoV) Situation Report - 121 January 2020. WHO Bull. 2020; (January): 1-7.

3. Lu R, Zhao X, Li J, Niu P, Yang B, Wu H, et al. Genomic characterisation and epidemiology of 2019 novel coronavirus: implications for virus origins and receptor binding. Lancet [Internet]. 2020; 395(10224): 565-74.

4. Rosenbaum L. Facing Covid-19 in Italy - Ethics, Logistics, and Therapeutics on the Epidemic's Front Line. N Engl J Med. 2020;1-3.

5. Chan-yeung M, Xu R. SARS: Epidemiology. Respirology. 2003; 8: S9-14.

6. Hellewell J, Abbott S, Gimma A, Bosse NI, Jarvis CI, Russell TW, et al. Feasibility of controlling COVID-19 outbreaks by isolation of cases and contacts. Lancet Glob Heal. 2020; 8(4): e488-96. 